\title{
The Critical Space for Orthogonally Invariant Varieties
}

\author{
Giorgio Ottaviani ${ }^{1}$
}

Received: 30 April 2021 / Accepted: 22 October 2021/Published online: 10 February 2022

(C) The Author(s) 2022

\begin{abstract}
Let $q$ be a nondegenerate quadratic form on $V$. Let $X \subset V$ be invariant for the action of a Lie group $G$ contained in $S O(V, q)$. For any $f \in V$ consider the function $d_{f}$ from $X$ to $\mathbb{C}$ defined by $d_{f}(x)=q(f-x)$. We show that the critical points of $d_{f}$ lie in the subspace orthogonal to $\mathfrak{g} \cdot f$, that we call critical space. In particular any closest point to $f$ in $X$ lie in the critical space. This construction applies to singular t-ples for tensors and to flag varieties and generalizes a previous result of Draisma, Tocino and the author. As an application, we compute the Euclidean Distance degree of a complete flag variety.
\end{abstract}

Keywords Tensors · Singular t-ples · Critical space $\cdot$ EDdegree $\cdot$ Euclidean Distance degree

Mathematics Subject Classification (2010) 14N07 - 14L30 - 14N05 · 15A69 - 15A18

\section{Introduction and Main Result}

Let $V$ be a complex vector space equipped with a nondegenerate symmetric bilinear form $q \in \operatorname{Sym}^{2} V$, identified in this paper with its associated quadratic form. The orthogonal group $S O(V, q)=S O(V)$ consists of linear transformations of $V$ leaving $q$ invariant. Let $X \subset V$ be an algebraic variety defined over $\mathbb{R}$, this includes the case when $X$ is the cone over a projective variety defined over $\mathbb{R}$. We assume that $X$ is $G$-invariant for the action of a Lie group $G \subset S O(V)$. In many cases of interest $X$ is $H$-invariant for a larger group $H$ and we can take $G=S O(V) \cap H$, see Section 2 for the case of partially symmetric tensors.

We denote by $\mathfrak{g}=T_{e} G$ the Lie algebra of $G$, where $e$ is the identity element, note that $\mathfrak{g} \subset \mathfrak{s o}(V)$. The tangent space to the orbit $G \cdot f$ at $f$ is $f+\mathfrak{g} \cdot f$. Denoting by $G_{f}=\{g \in G \mid g \cdot f=f\}$ the isotropy group of $f$, we have $\operatorname{dim} \mathfrak{g} \cdot f=\operatorname{dim} \mathfrak{g}-\operatorname{dim} G_{f}$.

Dedicated to Bernd Sturmfels on the occasion of his 60th birthday.

Giorgio Ottaviani

giorgio.ottaviani@unifi.it

1 Dipartimento di Matematica e Informatica "Ulisse Dini”, University of Florence, viale Morgagni 67/A, I-50134, Florence, Italy 
We define for $f \in V$ the critical space of $f$ as the subspace

$$
H_{f}:=(\mathfrak{g} \cdot f)^{\perp}=\{v \in V \mid q(v, w)=0 \quad \forall w \in \mathfrak{g} \cdot f\} .
$$

We remark that codim $H_{f}=\operatorname{dim} \mathfrak{g} \cdot f$, so we have codim $H_{f} \leq \operatorname{dim} \mathfrak{g}$ and the equality holds for general $f$ in many cases, but it cannot hold in the cases when $\operatorname{dim} \mathfrak{g} \geq \operatorname{dim} V$ (this happens when $V=\mathbb{C}^{a} \otimes \mathbb{C}^{b} \otimes \mathbb{C}^{c}$ and $c$ is large, in the setting of Section 2.2). Consider the function $d_{f}: X \rightarrow \mathbb{C}, d_{f}(x)=q(f-x, f-x)$, which, in the case $f$ is real, extends the squared distance function from $f$ defined over $\mathbb{R}$.

Note that at a critical point $x$ of $d_{f}$ we have $f-x \in\left(T_{x} X\right)^{\perp}$.

Lemma 1.1 If $g \in \mathfrak{g}$ then $q(g \cdot x, y)=-q(x, g \cdot y)$, in particular $q(g \cdot x, x)=0$.

Proof If $\mathfrak{g}=0$ then the statement is trivial. If $\mathfrak{g} \neq 0$, let $g(t) \subset G$ be a path such that $g(0)=$ $e$ and $\dot{g}(0)=g$. Taking the derivative at $t=0$ of the constant function $q(g(t) \cdot x, g(t) \cdot y)$ the thesis follows.

Our main result is the following theorem. Its proof is quite simple, nevertheless we will see in the rest of the paper it has some nontrivial consequences.

Theorem 1.2 Let $X$ be $G$-invariant for the action of $G \subset S O(V)$.

1. The critical points of $d_{f}$ on $X$ lie in $H_{f}$.

2. When $f$ is real, any closest point to $f$ in $X_{\mathbb{R}}$ (with respect to $q$ ) belongs to $H_{f}$.

3. $f \in H_{f}$.

Proof Let $x$ be a critical point. We need to prove $q(x-f, g \cdot f)=0 \forall g \in \mathfrak{g}$. We have $q(g \cdot f, f)=0 \quad \forall g \in \mathfrak{g}$ from Lemma 1.1. So it is enough to show that $q(x, g \cdot f)=0$ $\forall g \in \mathfrak{g}$. The crucial remark is that since $X$ is $G$-invariant then $\mathfrak{g} \cdot x \subset T_{x} X$. Since $x$ is critical it follows the chain of equalities (the second and the third one by Lemma 1.1 $0=q(g \cdot x, x-f)=-q(g \cdot x, f)=q(x, g \cdot f)$, which proves $(1)$.

(2) is an immediate consequence of (1).

(3) follows by $q(f, g \cdot f)=0$.

A partial converse to Theorem 1.2 is the following.

Theorem 1.3 Let $X$ be $G$-invariant for the action of $G \subset S O(V)$. Let $x \in H_{f} \cap X$.

1. If the orbit $G \cdot x$ is dense in $X$ then $x$ is a critical point of $d_{f}$ restricted to $X$.

2. If $X$ is a cone, $x$ is not isotropic and the orbit $G \cdot[x]$ is dense in $\mathbb{P} X$ then there is $\lambda \in \mathbb{C}$ such that $\lambda x$ is a critical point of $d_{f}$ restricted to $X$.

Proof We have the equality $\mathfrak{g} \cdot x=T_{x} X$ by assumption, and with this equality all the steps of the proof of Theorem 1.2 are invertible. This proves (1). The assumption of (2) implies that $\mathfrak{g} \cdot x+\langle x\rangle=T_{x} X$. Since $x$ is not isotropic there is $\lambda$ such that since $q(\lambda x, \lambda x-f)=0$, namely $\lambda=\frac{q(x, f)}{q(x, x)}$, so that orthogonality is guaranteed on the subspace $\langle x\rangle \subset T_{x} X$. To check orthogonality on the remaining part of $T_{x} X$ we may replace $x$ with $\frac{q(x, f)}{q(x, x)} x$ and the same argument in (1) works.

A stronger converse form will be proved for tensors, see Theorems 2.2 and 2.4 and for Grassmann varieties, see Theorem 3.1. In Theorem 3.4 we will compute the Euclidean 
Distance degree (EDdegree) of a complete flag variety with respect to the Frobenius product.

We recall that EDdegree $(X)$ (introduced in [4] by following an idea by Bernd Sturmfels) is the number of critical points of $d_{f}$ restricted to $X$ for general $f$. In many cases of interest it happens that $H_{f} \cap X$ is finite and reduced for general $f$, in these cases its cardinality counts EDdegree $(X)$, see (2.4) and Theorem 3.4.

The critical space was introduced for tensors in [14] and for partially symmetric tensors in [5], see also [15]. In Corollary 2.6 we get an alternative proof of the fact proved in [5] by Draisma, Tocino and the author that any best rank $q$ approximation of a partially symmetric tensor $f$ lies in the critical space.

Our approach is somehow dual to the one in $[3,6]$, where EDdegree was considered in an orthogonally invariant setting, but certain subvarieties of $X$ were constructed in order to cut transversally the orbits.

\section{Symmetric and Partially Symmetric Tensors}

\subsection{Symmetric Tensors}

Let $W$ a space of dimension $n+1$ and $V=\operatorname{Sym}^{d} W$. We assume that $W$ is equipped with a nondegenerate quadratic form $q_{W}$ and we choose coordinates in $W$ such that $q_{W}=$ $\sum_{i=0}^{n} x_{i}^{2}$. There is a unique nondegenerate bilinear form $q$ such that

$$
q\left(x^{d}, y^{d}\right)=q_{W}(x, y)^{d} \quad \forall x, y \in W,
$$

which is called the Frobenius (or Bombieri-Weyl) form. Since every polynomial in $\mathrm{Sym}^{d} W$ can be written as a sum of powers of linear forms, it is enough to ask (2.1) for any power $x^{d}, y^{d}$. The group $G=S O\left(W, q_{W}\right)$ acts over $V$ by the analogous rule $g \cdot\left(x^{d}\right)=$ $(g \cdot x)^{d}$. We get the inclusion $G \subset S O(V, q)$, so that we are in the setting of Section 1 ; our aim is to apply Theorem 1.2. The Frobenius form has the coordinate expression $q\left(\sum_{\alpha}\left(\begin{array}{l}d \\ \alpha\end{array}\right) f_{\alpha} x^{\alpha}, \sum_{\alpha}\left(\begin{array}{l}d \\ \alpha\end{array}\right) g_{\alpha} x^{\alpha}\right)=\sum_{\alpha}\left(\begin{array}{l}d \\ \alpha\end{array}\right) f_{\alpha} g_{\alpha}$ which, up to a scalar factor, has the nice M2 [10] implementation

$\operatorname{diff}(f, g)$.

Note that $S L(W) \cap S O(V)=S O(W)$, but we will not need this fact. The monomials are orthogonal but not orthonormal with respect to $q$.

\section{Proposition 2.1}

$$
\mathfrak{s o}(W) \cdot f=\left\langle x_{j} \frac{\partial f}{\partial x_{i}}-x_{i} \frac{\partial f}{\partial x_{j}}\right\rangle_{0 \leq i<j \leq n} .
$$

Proof It is convenient to denote

$$
D_{i j}(f)=x_{j} \frac{\partial f}{\partial x_{i}}-x_{i} \frac{\partial f}{\partial x_{j}} .
$$

For any skew-symmetric matrix $A$ we have that $e^{A}$ is orthogonal. Then $f\left(e^{t A} x\right)$ is a path in the $S O$-orbit of $f$. By taking the derivative at $t=0$ we get $\sum_{p=0}^{n} \frac{\partial f}{\partial x_{p}}(A x)_{p} \in \mathfrak{s o}(W) \cdot f$. By taking $A=e_{i j}-e_{j i}$ we get exactly $D_{i j} f$ and these elements $\operatorname{span} \mathfrak{s o}(W) \cdot f$. 
The rank one tensors in $\mathrm{Sym}^{d} W$ have the form $x^{d}$ and make a cone over the Veronese variety $v_{d} \mathbb{P} W$, where the origin has been removed from the cone. We recall that the eigenvectors of $f \in \operatorname{Sym}^{d} W$ are the critical points of the function $d_{f}\left(x^{d}\right)=q\left(f-x^{d}\right)$ restricted to the rank one tensors $[11,12,16,17,19]$. In this paper we are interested in the condition $x^{d} \in H_{f}$, which does not distinguish between $x$ and its scalar multiples, so by abuse of notation we may shift to projective space $\mathbb{P} W$ and denote by the same symbol the point $x \in \mathbb{P} W$. The eigenvectors correspond to the non isotropic $x$ (i.e. $q(x) \neq 0$ ) such that $\nabla f(x)=x$ in $\mathbb{P} W$, which means that any representatives of the right and the left hand side differ by a nonzero scalar multiple. The connection with (2.2) and (2.3) is that the eigenvectors of $f$ make the base locus of the linear system $\left\langle D_{i j} f\right\rangle$.

It follows from Theorem 1.2 that the eigenvectors of $f$ lie in $H_{f}$ (which is obvious from the above description since $D_{i j} f$ are the minors of the matrix $\left(\begin{array}{c}\nabla f \\ x\end{array}\right)$ ) and moreover the critical points of $d_{f}$ on the secant varieties of $d$-Veronese variety lie in $H_{f}$, which is not obvious from the definition and it was proved first in [5, Theorem 1.1]. We will state more precisely this claim in the more general setting of partially symmetric tensors in Corollary 2.6.

We give now a more precise converse to Theorem 1.2(1) in the case when $X$ is the cone of symmetric tensors of rank one.

Theorem 2.2 For general $f \in \operatorname{Sym}^{d} W, H_{f} \cap v_{d} \mathbb{P} W$ consists exactly of the critical points of $d_{f}$ restricted to $v_{d} \mathbb{P} W$, namely of the eigenvectors of $f$.

Proof Let $v^{d} \in H_{f} \cap v_{d} \mathbb{P} W$. In particular $q\left(v^{d}, g \cdot f\right)=0$ for any $g \in \mathfrak{g}$, which implies that $D_{i j} f$ vanishes at $v$. This is equivalent to the matrix

$$
\left(\begin{array}{c}
\nabla f \\
x
\end{array}\right)
$$

having rank one at $v$, which is the condition that $v$ is eigenvector of $f$, if $v$ is not isotropic. By [6, Lemma 4.2] the critical points of $d_{f}$ for a general $f$ avoid any proper closed subset of $v_{d} \mathbb{P} W$, so for general $f$ it is guaranteed that no isotropic $v$ is found.

Remark 2.3 Note that for even $d, \mathfrak{g} \cdot\left(f+c q^{d / 2}\right)=\mathfrak{g} \cdot f+\left[q^{d / 2}\right]$ for any $c \in \mathbb{C} \backslash\{0\}$. Conversely, if $\mathfrak{g} \cdot f=\mathfrak{g} \cdot h$ for general $f, h$ then we get $H_{f}=H_{h}$, so that $f, h$ have the same eigenvectors and Turatti proves in [20] (generalizing previous results from [1,2]) that there exists $c \in \mathbb{C}$ such that $f+c q^{d / 2}=h$.

\subsection{Partially Symmetric Tensors}

Consider the tensor product $\operatorname{Sym}^{d_{1}} V_{1} \otimes \cdots \otimes \operatorname{Sym}^{d_{k}} V_{k}=V$. We assume we have nondegenerate symmetric bilinear forms $q_{i}$ on $V_{i} . V$ is equipped with the Frobenius form $q$ such that on decomposable elements

$$
q\left(v_{1}^{d_{1}} \otimes \cdots \otimes v_{k}^{d_{k}}, w_{1}^{d_{1}} \otimes \cdots \otimes w_{k}^{d_{k}}\right)=\prod_{i=1}^{k} q_{i}\left(v_{i}, w_{i}\right)^{d_{i}} .
$$

The decomposable elements make a cone over the Segre-Veronese variety $X \simeq \mathbb{P} V_{1} \times \cdots \times$ $\mathbb{P} V_{k}$ embedded in $\mathbb{P} V$ with the line bundle $\mathcal{O}\left(d_{1}, \ldots, d_{k}\right)$. The group $G=S O\left(V_{1}, q_{1}\right) \times$ $\cdots \times S O\left(V_{k}, q_{k}\right)$ acts on $V$, we have again the inclusion $G \subset S O(V, q)$ and Theorem 1.2 
applies. Denote by $x_{i, 0} \ldots x_{i, n_{i}}$ an orthogonal coordinate system on $V_{i}$. Analogously to Proposition 2.1 the orbit $\mathfrak{s o}\left(V_{1}\right) \times \cdots \times \mathfrak{s o}\left(V_{k}\right) \cdot f$ is spanned by $x_{p, j} \frac{\partial f}{\partial x_{p, i}}-x_{p, i} \frac{\partial f}{\partial x_{p, j}}$ for $0 \leq i<j \leq n_{p}, p=1, \ldots, k$. It follows that the critical space $H_{f}$ defined according to (1.1) coincides with the one defined in [5].

The critical points of $d_{f}(x)=q(f-x)$ restricted to the Segre-Veronese variety are the singular t-ples of $f$ [12], their number is called EDdegree in [4] and it is counted by the formula in [7], see also [4, §8].

The proof of Theorem 2.2 generalizes to this setting and gives

Theorem 2.4 For general $f \in \operatorname{Sym}^{d_{1}} V_{1} \otimes \cdots \otimes \operatorname{Sym}^{d_{k}} V_{k}=V$, let $X \subset \mathbb{P} V$ be the Segre-Veronese variety of rank one tensors. $H_{f} \cap X$ consists exactly of the singular $t$-ples of $f$.

Since general partially symmetric tensors $f$ have trivial isotropic groups, in the binary case $X_{\mathbf{d}}=\mathbb{P}^{1} \times \cdots \times \mathbb{P}^{1}$ embedded in $\mathbb{P}\left(\operatorname{Sym}^{d_{1}} \mathbb{C}^{2} \otimes \cdots \otimes \operatorname{Sym}^{d_{k}} \mathbb{C}^{2}\right)$ with the line bundle $\mathcal{O}\left(d_{1}, \ldots, d_{k}\right)$ we have $G=\left(\mathbb{C}^{*}\right)^{k}, \mathfrak{g}=\mathbb{C}^{k}$ and the nice coincidence codim $H_{f}=k=$ $\operatorname{dim} X_{\mathbf{d}}$. Hence the cardinality of the intersection between $H_{f}$ and $X_{\mathbf{d}}$ can be counted by Bezout Theorem and it follows an alternative proof of the formula

$$
\operatorname{EDdegree}\left(X_{\mathbf{d}}\right)=\operatorname{deg} X_{\mathbf{d}}=k ! d_{1} \ldots d_{k},
$$

already known from [7], [18, Eq. (1.6)]. Our approach explains that the resulting equality between EDdegree and deg of $X_{\mathbf{d}}$ is not a coincidence. Note that Bezout Theorem applies when the intersection scheme has the expected codimension, without assuming $H_{f}$ being general, see (1) in $[8, \S 8.4]$. We will apply again this approach to complete flag varieties in Theorem 3.4.

Example 2.5 If $\operatorname{dim} A=\operatorname{dim} B=\operatorname{dim} C=2$ we denote by $Q_{A}$, (resp. $Q_{B}, Q_{C}$ ) the isotropic quadric consisting of two points on $\mathbb{P}(A)$ (resp. $\mathbb{P}(B), \mathbb{P}(C)$ ).

We have that $\operatorname{dim}(\mathfrak{s o} \cdot f)<3$ if and only if $f$ belongs to one of the following six $\mathbb{P}^{3}$ linearly embedded in $\mathbb{P}(A \otimes B \otimes C)$ (each item consists of two $\mathbb{P}^{3}$ 's)

$$
Q_{A} \times \mathbb{P}(B \otimes C), \quad Q_{B} \times \mathbb{P}(A \otimes C), \quad Q_{C} \times \mathbb{P}(A \otimes B) .
$$

The following result was proved in [5], joint with J. Draisma and A. Tocino. The proof given here, as a consequence of Theorem 1.2, is maybe simpler.

Corollary 2.6 [5, Theorem 1.1] Let $X_{q}$ be the q-secant variety to the Segre-Veronese variety in $\mathbb{P}\left(\operatorname{Sym}^{d_{1}} V_{1} \otimes \cdots \otimes \operatorname{Sym}^{d_{k}} V_{k}\right)$. Then the critical points of the distance function from a tensor $f$ to $X_{q}$ lie in $H_{f}$. In particular any best rank $q$ approximation of $f$ (when it exists) lie in $H_{f}$.

\section{Grassmann and Flag Varieties}

\subsection{Grassmann Varieties}

Let $V=\wedge^{k} W$, we consider the Grassmann variety $\operatorname{Gr}(k, W)$ of $k$-dimensional subspaces of $W$, its cone is embedded in $V$. Again, a nondegenerate quadratic form $q_{W}$ on $W$ extends to the Frobenius form $q$ on $V$ by requiring $q\left(v_{1} \wedge \cdots \wedge v_{k}, w_{1} \wedge \cdots \wedge w_{k}\right)=\operatorname{det}\left(q_{W}\left(v_{i}, w_{j}\right)\right)$ 
(the Gram determinant). If $v=v_{1} \wedge \cdots \wedge v_{k} \in \wedge^{k} W$ then the derivative $\frac{\partial v}{\partial x_{i}} \in \wedge^{k-1} W$ is defined by the Leibniz formula

$$
\frac{\partial v}{\partial x_{i}}=\sum_{j=1}^{k} v_{1} \wedge \cdots \wedge \frac{\partial v_{j}}{\partial x_{i}} \wedge \cdots \wedge v_{d}
$$

and extended by linearity to all $\wedge^{k} W$. This is compatible with the inclusion $\wedge^{k} W \subset W^{\otimes k}$ and the form $q$ just defined is the restriction of the Frobenius form on $W^{\otimes k}$ of the previous section. The same formula (2.2) holds formally in case $S O(W)$ acts on $\wedge{ }^{k} W$.

$$
\mathfrak{s o}(W) \cdot f=\left\langle\frac{\partial f}{\partial x_{i}} \wedge x_{j}-\frac{\partial f}{\partial x_{j}} \wedge x_{i}\right\rangle_{0 \leq i<j \leq n} .
$$

The EDdegree of Grassmann varieties with respect to the Frobenius form is still unknown in general.

For a general $f \in \wedge^{k} W$, we have that a non isotropic $v=v_{1} \wedge \cdots \wedge v_{k}$ is a critical point for $d_{f}$ if $T\left(v_{1} \wedge \cdots \widehat{v_{i}} \cdots \wedge v_{k}\right)=q\left(v_{i},-\right) \forall i=1, \ldots k$. Again, the proof of Theorem 2.2 generalizes to this setting and gives

Theorem 3.1 For general $f \in \wedge^{k} W, H_{f} \cap G r(k, W)$ consists exactly of the critical points of $d_{f}$ restricted to the Grassmann variety $G r(k, W)$.

\subsection{Flag Varieties}

For a flag variety $X=S L(W) / P$, where $P$ is a parabolic subgroup of $S L(W)[9, \S 23.3]$, embedded by a very ample line bundle $L, H_{f} \cap X$ consists exactly of the critical points of $d_{f}$. The embedding space is a Schur module $S^{\alpha} W$ where the Frobenius form is defined again by restriction of the one on $W^{\otimes k}$ and again we have $G=S O(W)$.

For complete flag varieties $\mathbb{F}_{n}$, which parametrize complete flags $\left(L_{1} \subset \cdots \subset L_{n}\right) \subset W$ with $\operatorname{dim} L_{i}=i$ (partial flags may miss some $L_{i}$ 's), the above principle becomes effective in computing the number of critical points. We recall that $\operatorname{dim} \mathbb{F}_{n}=n(n+1) / 2$ and that $\mathbb{F}_{n}=$ $S L(n+1) / B$ where $B$ is the Borel subgroup of upper triangular matrices. The following two lemmas are well known, we include the proofs for the convenience of the reader.

Lemma $3.2 \chi\left(\mathbb{F}_{n}, \mathbf{Z}\right)=(n+1)$ !.

Proof A general section of the tangent bundle $T \mathbb{F}_{n}$ is given by a matrix $A \in S L(n+$ $1)=S L(W)$ with distinct eigenvalues and corresponding eigenvectors $v_{1}, \ldots v_{n+1}$. The zero locus of this section consists of $A$-invariant complete flags $\left(L_{1} \subset \ldots \subset L_{n}\right)$ with $\operatorname{dim} L_{i}=i$. There are $(n+1)$ choices for $L_{n}$, obtained by the span of $n$ among the $v_{i}$. For each $L_{n}$ there are correspondingly $n$ choices for $L_{n-1}$, and so on there are $(n+1)$ ! choices for each $A$-invariant complete flag. The thesis follows from Gauss-Bonnet theorem.

Lemma 3.3 (i) Let $\mathbb{F}_{n}$ be embedded with the line bundle $\mathcal{O}\left(a_{1}, \ldots, a_{n}\right)$ in the projective space over $S^{a_{1}, \ldots, a_{n}} \mathbb{C}^{n+1}$, the module with Young diagram having $\sum_{i=j}^{n} a_{i}$ boxes in the jth row. The degree of the embedded variety is

$$
\left(\begin{array}{c}
n+1 \\
2
\end{array}\right) ! \prod_{1 \leq i<j \leq n+1} \frac{a_{i}+\cdots+a_{j-1}}{j-i} .
$$

(ii) When $a_{i}=1$ we get $\operatorname{deg} \mathbb{F}_{n}=\left(\begin{array}{c}n+1 \\ 2\end{array}\right)$ !. 
Proof We have $H^{0}\left(\mathbb{F}_{n}, \mathcal{O}\left(a_{1}, \ldots, a_{n}\right)\right)=\prod_{1 \leq i<j \leq n+1} \frac{a_{i}+\cdots+a_{j-1}+j-i}{j-i}$ by Weyl character formula (see [9, equation (15.17)]). Then the Hilbert polynomial is $H^{0}\left(\mathbb{F}_{n}, \mathcal{O}\left(t a_{1}, \ldots, t a_{n}\right)\right)=\prod_{1 \leq i<j \leq n+1} \frac{t\left(a_{i}+\cdots+a_{j-1}\right)+j-i}{j-i}$ and computing the leading term we get the thesis. In case (ii) the Hilbert polynomial simplifies to $\chi\left(\mathbb{F}_{n}, \mathcal{O}(t, \ldots, t)\right)=$

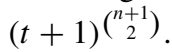

Theorem 3.4 Let $B \subset S L(n+1)$ be the Borel subgroup of upper triangular matrices. For a complete flag variety $\mathbb{F}_{n}=S L(n+1) / B$, embedded by a very ample line bundle $L=\mathcal{O}\left(a_{1}, \ldots, a_{n}\right)$, with respect to the Frobenius form we have that EDdegree $\mathbb{F}_{n}=\operatorname{deg} \mathbb{F}_{n}$ is given by (3.2).

Proof For general $f \in H^{0}(S L(n+1) / B)$, we have again the nice coincidence that the codimension of $H_{f}$ is equal to the dimension of $\mathbb{F}_{n}$, which is $\left(\begin{array}{c}n+1 \\ 2\end{array}\right)=\operatorname{dim} S O(n+1)$, so that the critical points are cut by a linear space of complementary dimension.

Example 3.5 For $n=2$, the flag variety $S L(3) / B$ (see I $\S 3.1$ of [13]) embedded with $\mathcal{O}(a, b)$ has EDdegree $\mathbb{F}_{2}=\operatorname{deg} \mathbb{F}_{2}=3 a b(a+b)$.

Acknowledgements The author is member of Italian GNSAGA-INDAM. Partially supported by the H2020MSCA-ITN-2018 project POEMA.

Open Access This article is licensed under a Creative Commons Attribution 4.0 International License, which permits use, sharing, adaptation, distribution and reproduction in any medium or format, as long as you give appropriate credit to the original author(s) and the source, provide a link to the Creative Commons licence, and indicate if changes were made. The images or other third party material in this article are included in the article's Creative Commons licence, unless indicated otherwise in a credit line to the material. If material is not included in the article's Creative Commons licence and your intended use is not permitted by statutory regulation or exceeds the permitted use, you will need to obtain permission directly from the copyright holder. To view a copy of this licence, visit http://creativecommons.org/licenses/by/4.0/.

\section{References}

1. Abo, H., Seigal, A., Sturmfels, B.: Eigenconfigurations of Tensors. In: Harrington, H.A., Omar, M., Wright, M. (eds.) Algebraic and Geometric Methods in Discrete Mathematics. Contemporary Mathematics, vol. 685, pp. 1-25. AMS, Providence (2017)

2. Beorchia, V., Galuppi, F., Venturello, L.: Eigenschemes of ternary tensors. SIAM J. Appl. Algebra Geom. 5, 620-650 (2021)

3. Bik, A., Draisma, J.: A note on ED degrees of group-stable subvarieties in polar representations. Isr. J. Math. 228, 353-377 (2018)

4. Draisma, J., Horobeţ, E., Ottaviani, G., Sturmfels, B., Thomas, R.: The Euclidean distance degree of an algebraic variety. Found. Comput. Math. 16, 99-149 (2016)

5. Draisma, J., Ottaviani, G., Tocino, A.: Best rank- $k$ approximation for tensors: generalizing EckartYoung. Res. Math. Sci. 5, 27 (2018)

6. Drusvyatskiy, D., Lee, H.-L., Ottaviani, G., Thomas, R.: The Euclidean distance degree of orthogonally invariant matrix varieties. Isr. J. Math. 221, 291-316 (2017)

7. Friedland, S., Ottaviani, G.: The number of singular vector tuples and uniqueness of best rank-one approximation of tensors. Found. Comput. Math. 14, 1209-1242 (2014)

8. Fulton, W.: Intersection Theory. Springer, Berlin (1984)

9. Fulton, W., Harris, J.: Representation Theory. Springer, New York (1991)

10. Grayson, D., Stillman, M.: Macaulay2, a software system for research in Algebraic Geometry. https:// faculty.math.illinois.edu/Macaulay2/. http://www2.macaulay2.com/Macaulay2/Citing/

11. Hu, S., Qi, L.: The E-eigenvectors of tensors. Linear Multilinear Algebra 62, 1388-1402 (2014) 
12. Lim, L.-H.: Singular values and eigenvalues of tensors: a variational approach. In: Proc. IEEE Int. Workshop on Comput. Advances in Multi-Sensor Adaptive Processing (CAMSAP 2005), pp. 129-132. IEEE (2005)

13. Okonek, C., Schneider, M., Spindler, H.: Vector Bundles on Complex Projective Spaces. Progress in Mathematics, vol. 3. Birkhäuser, Boston (1980)

14. Ottaviani, G., Paoletti, R.: A geometric perspective on the singular value decomposition. Rend. Istit. Mat. Univ. Trieste 47, 107-125 (2015)

15. Ottaviani, G., Sodomaco, L., Ventura, E.: Asymptotics of degrees and ED degrees of Segre products. Adv. Appl. Math. 130, 102242 (2021)

16. Qi, L.: Eigenvalues of a real supersymmetric tensor. J. Symb. Comput. 40, 1302-1324 (2005)

17. Qi, L., Luo, Z.: Tensor Analysis: Spectral Theory and Special Tensors. SIAM, Philadelphia (2017)

18. Sodomaco, L.: On the product of the singular values of a binary tensor. Isr. J. Math. 243, 233-272 (2021)

19. Sturmfels, B.: Tensors and their eigenvectors. Not. Amer. Math. Soc. 63, 604-606 (2016)

20. Turatti, E.: On tensors which are determined by their singular tuples. arXiv:2104.03686 (2021)

Publisher's Note Springer Nature remains neutral with regard to jurisdictional claims in published maps and institutional affiliations. 